\title{
INDICADOR SINTÉTICO PARA LA MEDICIÓN DE LA NECESIDAD DE INVESTIGACIÓN Y GESTIÓN AMBIENTAL BASADO EN MORBIMORTALIDAD OCURRIDA EN 2009-2012, META, COLOMBIA
}

\author{
Oscar Alexander Gutiérrez Lesmes ${ }^{1}$ \\ Claudia Marcela Martínez Agudelo ${ }^{2}$
}

Recibido el 8 de febrero de 2015, aprobado el 17 de junio de 2015 y actualizado el 03 noviembre de 2015

DOI: 10.17151/luaz.206.42.10

\section{RESUMEN}

Objetivo: Construir un indicador sintético basado en degradación en salud en los municipios del departamento del Meta en el periodo 2009-2012, que permita priorizar las necesidades de investigación e intervención ambiental de estos municipios. Metodología: Se realizó un estudio analítico, observacional, exploratorio, retrospectivo. Los datos se obtuvieron de las base de datos estatales de vigilancia en salud pública y el registro único de afiliados (RUAF), de 2009 a 2012 del departamento del Meta. Una vez fueron seleccionados los casos de análisis se construyeron nuevas bases de datos, las cuales fueron analizadas en 4 fases: construcción de los indicadores simples, índices temáticos, los indicadores compuestos, y el indicador sintético global. Resultados: El indicador arrojó un valor para cada uno de los 29 municipios, evidenciando diferencias en los efectos ambientales sobre la salud. San Carlos de Guaroa con el mayor puntaje (10200) y Puerto Concordia (1000). Al estratificar el indicador lo municipios clasificaron así: necesidad alta, necesidad media, necesidad baja: Discusión: Considerar a la salud como un indicador del estado del ambiente y al ambiente como determinante causal de la salud ha generado el desarrollo de diferentes metodologías para medir la relación causa-efecto entre el ambiente y la salud. Se infiere la presencia de factores ambientales desencadenantes de la morbimortalidad, reconocidos por la OMS como fuerza impulsora, presión o de exposición en la causalidad de origen ambiental (Yassi et al., 2002; Posada et al., 2004), responsables de los diferentes comportamientos en la degradación en salud. Conclusiones: El indicador permite medir las diferencias ambientales entre municipios, las diferencias en los efectos del ambiente sobre la salud, permiten inferir las condiciones ambientales de los municipios, se observan la presencia de efectos provenientes de: el agua, el aire, sustancias químicas, fauna.

\section{PALABRAS CLAVE}

Indicadores, medio ambiente y salud pública, salud ambiental, factores epidemiológicos.

\section{SYNTHETIC INDICATOR FOR MEASURING THE NEED FOR RESEARCH AND ENVIRONMENTAL MANAGEMENT BASED ON MORBIMORTALITY IN THE PERIOD 2009-2012, IN META, COLOMBIA}

\begin{abstract}
Objective: To build a synthetic indicator based on health degradation in the municipalities of the Department of Meta in the period 2009-2012 to enable prioritizing research needs and environmental intervention in these municipalities. Methodology: An analytical, observational, exploratory, retrospective study was carried out. Data were obtained from the state public health surveillance databases and the unified affiliated record (UAR), in the period
\end{abstract}


2009-2012 in the Department of Meta. Once the cases for analysis were selected, new databases were created which were analyzed in 4 stages: construction of simple indicators; thematic indexes; composite indicators, and the overall synthetic indicator. Results: The indicator showed a value for each of the 29 municipalities, making clear differences in environmental effects on health. San Carlos de Guaroa with the highest score $(10,200)$ and Puerto Concordia $(1,000)$. By stratifying the indicator, municipalities classified as: high need, average requirement, and low need. Discussion: Considering health as an indicator of the state of the environment and the environment as a determinant causal of health has generated the development of different methodologies to measure the cause-effect relationship between environment and health. The presence of environmental factors which trigger morbimortality, recognized by the World Health Organization (WHO) as a driving force, pressure or exposure in the causation of environmental origin (Yassi et al., 2002; Posada et al., 2004), responsible for different behaviors in health degradation is concluded. Conclusions: The indicator allows measuring environmental differences between municipalities, and the differences in the effects of environment on health, as well as inferring the environmental conditions of the municipalities buy observing the presence of effects from: water, air, chemical substances, and fauna.

KEY WORDS: Indicators, environment and public health, environmental health, epidemiologic factors.

\section{INTRODUCCIÓN}

Disponer de un indicador sintético para evaluar las condiciones ambientales en los municipios del departamento del Meta, constituye un avance significativo, permite la clasificación diferencial de estas condiciones entre municipios del departamento.

El ambiente genera el $24 \%$ de la morbilidad y el $25 \%$ de las muertes tempranas en el mundo, en los países en desarrollo el porcentaje de mortalidad atribuible a causas ambientales es del $25 \%$, y en desarrollados del 17\% (Prüss-Üstün y Corvalán, 2006) y en menores de 15 años se atribuye el $36 \%$ de la mortalidad a causas ambientales, esto es alrededor de la cuarta parte de la carga mundial de morbilidad. En América Latina se estima que más del 10\% de todos los años de vida perdidos por discapacidad se deben directamente a factores ambientales, correspondiéndole al abastecimiento de agua y saneamiento básico el 5,5\%, y el $4,5 \%$ restante a contaminación atmosférica urbana, sustancias químicas, desechos agroindustriales y contaminación del aire interior (Organización Panamericana de la Salud, 2001).

La interacción de los factores ambientales naturales y antrópicos con la población humana genera un comportamiento epidemiológico específico, en esta interacción cada factor ejerce un rol en la generación de causalidad para la presencia de morbimortalidad en una población. Según Ordóñez (2000), han descrito más de 64 determinantes ambientales que participan en la morbimortalidad conocida en el mundo, estos factores han sido clasificados en dos clases: naturales y antrópicos (Puente, 2008); por otro lado, la Organización Mundial de la Salud ha descrito 85 enfermedades con su respectiva mortalidad, las cuales califica como ocasionadas por determinantes ambientales (Prüss-Üstün y Corvalán, 2006), varias de estas enfermedades hacen parte del perfil epidemiológico del Meta.

El Ministerio de Salud y Protección Social en el documento dimensiones prioritarias en salud pública del Plan Decenal de Salud Pública 2012-2021 estima 
que: "el $41 \%$ de los colombianos viven en grandes ciudades en donde se encuentra la mayor concentración de contaminantes en el aire como PM10, PM $2,5, \mathrm{CO}, \mathrm{NO} 2, \mathrm{SO} 2$ y O3, y además podrían darse otros tipos de contaminantes tales como el ruido, campos electromagnéticos y olores ofensivos" (2012, p. 23), estas características pueden ser extrapoladas a algunas ciudades del departamento del Meta; otra característica de las ciudades colombianas que también está presente en el Meta es el bajo acceso al agua potable; el sistema de vigilancia a la calidad del agua en Colombia muestra que $52,9 \%$ de los municipios no disponen de agua apta para el consumo humano y solo el $25,4 \%$ de los municipios disponen de agua sin riesgo para el consumo humano (Instituto Nacional de Salud, 2007).

\section{METODOLOGÍA}

Se realizó un estudio analítico, observacional, exploratorio, retrospectivo. Los datos se obtuvieron de las base de datos estatales de vigilancia en salud pública (SIVIGILA) y el registro único de afiliados (RUAF), de los años 2009, 2010, 2011 y 2012 del departamento del Meta.

Las bases del SIVIGILA contenían el registro de 71.723 y las bases del RUAF 25.037 registros procedentes de atenciones médicas realizadas en los diferentes municipios del departamento del Meta, en los centros de salud, clínicas y hospitales de la red de Instituciones prestadoras de salud del Meta.

Las bases fueron filtradas mediante los siguientes criterios: la enfermedad o la causa de muerte estén clasificadas por la Organización Mundial de la Salud como morbimortalidad de causa ambiental (World Health Organization, 2004; Quezada, 2006), y el lugar de residencia de la persona seleccionada por el tipo de evento fuese un municipio del departamento del Meta. Los demás casos fueron retirados de la base de datos bajo los siguientes criterios de exclusión: la enfermedad o la causa de muerte no están clasificadas por la Organización Mundial de la Salud como morbimortalidad de causa ambiental y/o el municipio de residencia y/o procedencia donde ocurre la morbimortalidad no es del departamento del Meta.

Una vez fueron seleccionados los casos de análisis se construyeron nuevas bases de datos, las cuales fueron analizadas en 4 fases: construcción de los indicadores simples, índices temáticos, los indicadores compuestos, y el indicador sintético.

En la Fase 1, para cada municipio se construyó la incidencia acumulada y la tasa de mortalidad por evento ocurrida en el periodo 2009-2012; los eventos a los cuales se les estimó la incidencia acumulada y tasa la mortalidad fueron: intoxicación por metales, intoxicación por plaguicidas, intoxicación por solvente, intoxicación por otras sustancias químicas, accidente ofídico, agresiones por animales potencialmente transmisores de rabia, leptospirosis, enfermedad diarreica aguda global, dengue global, paludismo en todas sus formas, leishmaniasis, enfermedad de Chagas, fiebre amarilla, ESI (enfermedad similar a influenza), IRA (infección respiratoria aguda), IRAG (infección respiratoria aguda grave), IRAG inusitado.

En la construcción de la incidencia y de la tasa de mortalidad se utilizó como denominador la media de la población de cada municipio para el período según reporte del Departamento Nacional de Estadística. Todas las tasas fueron calculadas usando una constante de 100.000 habitantes. 
Para el cálculo de la incidencia acumulada (IA) de cada uno de los eventos se utilizó la siguiente fórmula:

Incidencia acumulada de $\left(\mathrm{a}^{*}\right)=\frac{\text { Número de enfermos por }\left(\mathrm{a}^{*}\right) \text { en un periodo y lugar }}{\text { Población susceptible en el periodo y lugar }} \times 100.000$
* Representa la morbilidad para la cual se calcula la incidencia.

Para el cálculo de la tasa de mortalidad (TM) de cada uno de los eventos se utilizó la siguiente fórmula:

Tasa de mortalidad por causas $=\frac{\text { Número de muertes por }\left(a^{*}\right) \text { en un periodo y lugar }}{\text { Población a mitad del período en el lugar }} \times 100.000$
* Representa la causa de muerte para la cual se calcula la tasa.

En la Fase 2 se construyeron índices temáticos no ponderados con las incidencias y con las tasas de mortalidad de la primera fase, para la construcción de los índices temáticos se utilizaron las siguientes formulas:

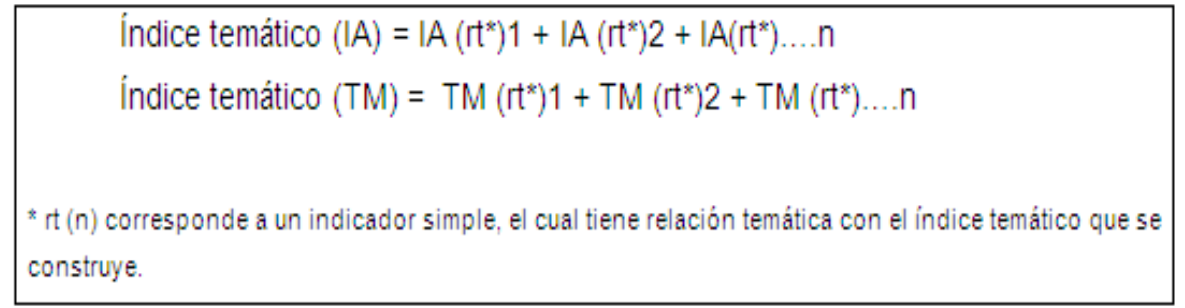

Cada índice temático es la agrupación de las incidencias o de tasas de mortalidad, basados en la teoría de la historia natural de la enfermedad, de esta manera se construyeron las siguientes agrupaciones: intoxicaciones químicas, zoonosis, infección gastrointestinal, enfermedades transmitidas por vectores e infecciones respiratorias.

En la Fase 3 se construyeron indicadores compuestos por municipio, en el marco teórico de indicadores de desarrollo sostenible del Decreto 1200 de 2004 del Ministerio de Ambiente, Vivienda y Desarrollo (Ministerio de Ambiente, Vivienda y Desarrollo, 2004) los cuales reconocen el impacto del ambiente derivado de la calidad del agua y la calidad del aire; estos indicadores buscan evidenciar el impacto de la gestión ambiental orientada hacia el desarrollo sostenible (Schuschny y Soto, 2009). Y en la metodología DPSEEA, la cual determina: (Fuerzas impulsoras $\rightarrow$ Presión $\rightarrow$ Estado $\rightarrow$ Exposición $\rightarrow$ Efecto, Acción) (Posada et al., 2004).

Estos índices no son medición del estado ambiental pero representan el efecto, y son propuestos para medir la necesidad de gestión ambiental en términos de estimar el estado de las condiciones ambientales y necesidades de intervención. 


\begin{tabular}{|l|}
\hline Indicador compuesto $=I T M^{*} 1+I T\left|A^{*} 1+\right| T M^{*} 2+I T I A^{*} 2 \ldots . n$ \\
$* I T M(n)$ corresponde a un índice temático de mortalidad clasificado en un factor ambiental. \\
*ITIA (n) corresponde a un índice temático de incidencia acumulada clasificado en un factor ambiental.
\end{tabular}

En la sumatoria de los casos de morbimortalidad dejaron de ser considerados casos para asumir el valor de puntos representados en una escala, los efectos sobre la salud fueron agrupados en cuatro grupos similares a los determinados por la OMS en la metodología DPSEE, obteniendo como resultado los cuatro indicadores sintéticos.

Una vez determinados los indicadores de la Fase 3 se clasificaron los municipios en tres categorías (baja, media y alta) en cada uno de los indicadores sintéticos, la clasificación se realizó mediante los percentiles 33,3 y 66,3. Una vez clasificados los municipios se reasignó un nuevo valor a cada municipio, según la categoría en la que habían sido clasificados, de la siguiente manera: baja: 33 , media: 66 , alta: 100.

En la Fase 4 se construyó el indicador sintético sumando los puntajes obtenidos por cada municipio en cada uno de los 4 indicadores compuestos, este puntaje final permitió estratificar de mayor a menor el gradiente de salud ambiental en los municipios del Meta, y con base en éste se priorizó la necesidad de medición e intervención ambiental para determinar causalidad y disminuir el impacto en la salud, este indicador fue denominado como la Necesidad de gestión ambiental, edición e intervención ambiental por degradación en salud.

El indicador sintético también se estratificó mediante percentiles para clasificar la necesidad de los municipios en alta, media y baja, estos resultados fueron aplicados en un mapa temático.

Indicador sintético global por municipio $=\sum$ (puntos por clasificación de los 4
indicadores compuestos)

\section{RESULTADOS}

En la Tabla 1 se observan los municipios y el valor obtenido en el indicador sintético (Necesidad de gestión ambiental, medición e intervención ambiental por degradación en salud), los cuales fueron clasificados del número 1 al 29 según el puntaje obtenido por cada uno de los municipios; todos los municipios obtienen un puntaje que evidencia la presencia de efectos del ambiente sobre el perfil epidemiológico.

El indicador permite sumar todos los efectos sobre la salud de diferentes morbilidades y mortalidades, evidenciando cuáles son los municipios que tienen la mayor acumulación de efectos.

En la clasificación se observan los 10 municipios con el puntaje más alto obtenido por degradación en salud por efecto de variables ambientales, en orden descendente fueron: San Carlos de Guaroa, Barranca de Upía, Cabuyaro, Acacías, Granada, Villavicencio, Restrepo, El Dorado, Cumaral, Castilla la Nueva, 
Puerto Gaitán. Debe tenerse en cuenta que todos los municipios presentan degradación en salud por factores ambientales.

\begin{tabular}{|c|c|c|}
\hline \multicolumn{3}{|c|}{ smbiental por degradación en salud } \\
\hline Municipio & indicador sintético & Clasificación \\
\hline San Carlos de Guaroa & 10200 & 1 \\
\hline Barranca de Upia & 10100 & 2 \\
\hline Cabuyaro & 9300 & 3 \\
\hline Acacias & 9200 & 4 \\
\hline Granada & 9200 & 5 \\
\hline Villavicencio & 9200 & 6 \\
\hline Restrepo & 8700 & 7 \\
\hline El Dorado & 8600 & 8 \\
\hline Cumaral & 8200 & 9 \\
\hline Castilla la Nueva & 8000 & 10 \\
\hline Puerto Gaitán & 7800 & 11 \\
\hline Fuentedeoro & 7200 & 12 \\
\hline Guamal & 7200 & 13 \\
\hline San Martín & 6700 & 14 \\
\hline Mapiripán & 6200 & 15 \\
\hline El Calvario & 6000 & 16 \\
\hline Puerto López & 5900 & 17 \\
\hline Puerto Lleras & 5800 & 18 \\
\hline Lejanias & 5500 & 19 \\
\hline El Castillo & 4900 & 20 \\
\hline Puerto Rico & 4700 & 21 \\
\hline San Juan de Arama & 4600 & 22 \\
\hline Cubarral & 4200 & 23 \\
\hline La Macarena & 4100 & 24 \\
\hline Mesetas & 4000 & 25 \\
\hline San Juanito & 3900 & 26 \\
\hline La Uribe & 3800 & 27 \\
\hline Vistahermosa & 1400 & 28 \\
\hline Puerto Concordia & 1000 & 29 \\
\hline
\end{tabular}

En la Figura 1 se observan los estratos construidos para el indicador sintético (Necesidad de gestión ambiental, medición e intervención ambiental por degradación en salud), estos estratos se pudieron construir ya que el indicador sintético permite crear grupos definidos mediantes percentiles, estos se aplicaron a los puntajes obtenidos en la sumatoria de los indicadores compuestos de la Fase 3.

Se observan las categorías alta, media y baja necesidad, en las que se clasifican los municipios. Los municipios se ubicaron de la siguiente manera: necesidad alta para San Carlos de Guaroa, Barranca de Upía, Cabuyaro, Acacías, Granada, Villavicencio, Restrepo, El Dorado, Cumaral, Castilla la Nueva. Los municipios clasificados como de necesidad media son: Puerto Gaitán, Fuentedeoro, Guamal, San Martín, Mapiripán, El Calvario, Puerto López, Puerto Lleras, Lejanías, y los municipios clasificados como de necesidad baja fueron: El Castillo, Puerto Rico, San Juan de Arama, Cubarral, La Macarena, Mesetas, San Juanito, La Uribe, Vistahermosa, Puerto Concordia. 
Los municipios con los resultados de necesidad de gestión ambiental más alta son Villavicencio (capital del departamento) y los municipios de su área metropolitana, sumado a estos el municipio de Granada, el cual es el segundo municipio en densidad poblacional y desarrollo del departamento.

Figura 1. Necesidad de gestión ambiental, medición e intervención ambiental por degradación en salud

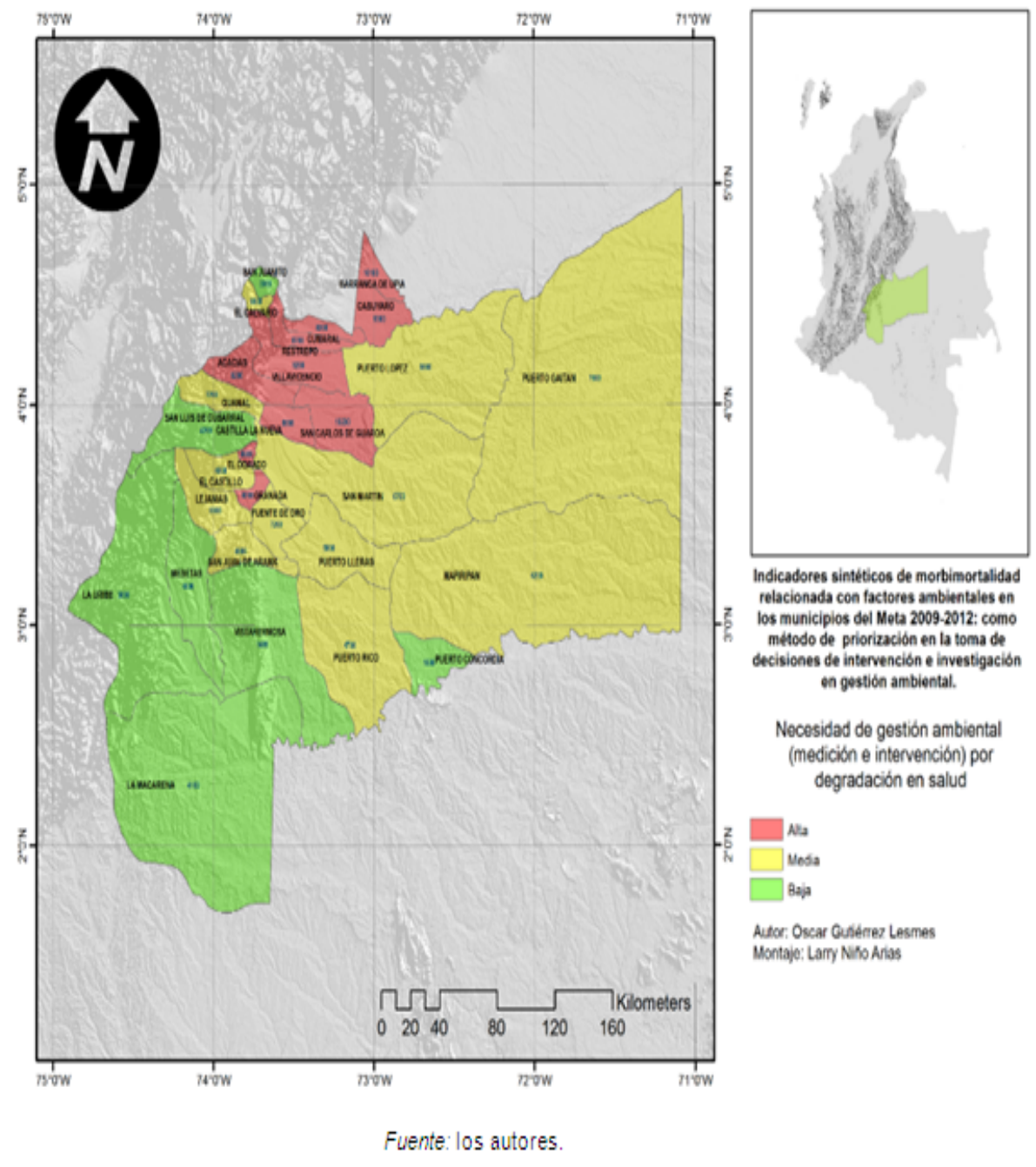

\section{DISCUSIÓN}

Considerar a la salud como un indicador del estado del ambiente y al ambiente como determinante causal de la salud ha generado esfuerzos en diferentes países en la búsqueda de la relación entre el ambiente y la salud, el trabajo de estos países ha desarrollado metodologías para realizar estas mediciones (Casas et al., 2011).

El impacto generado por determinantes ambientales en la calidad de vida y la salud humana son temáticas recientes en la evaluación del impacto ambiental en Colombia (Piña, 2010; García-Ubaque et al., 2012). Por otra parte, la salud 
ambiental como parte del área de la salud pública ha tenido por objeto la identificación, caracterización, vigilancia, control y evaluación de los efectos en la salud de los factores de riesgo ambientales (Posada et al., 2004; Pérez et al., 2011).

El proceso salud-enfermedad, enmarcado en el modelo de determinantes sociales, reconoce que los principales determinantes de la salud no proceden mayoritariamente ni directamente del sistema de salud sino del ambiente (ÁlvarezPérez et al., 2007; Villar, 2007). El nivel de salud de la población está condicionado por factores biológicos (ambiente), estilos de vida, clase social (ambiente), saneamiento básico (ambiente), medio ambiente (ambiente) y el acceso a servicios y el empleo (ambiente), la vivienda (ambiente) (Moiso y Barragán, 2007; Pérez et al., 2011).

Lo anterior y debido a que en el ambiente se encuentran agentes causales (naturales o antrópicos) de naturaleza física, química o biológica que pueden producir efectos adversos para la salud de la población (Ordóñez, 2000; Yassi et al., 2002) y los efectos de estos deben ser utilizados como trazadores de las condiciones ambientales (Ordóñez, 2000; Yassi et al., 2002; Posada et al., 2004).

Las metodologías más importantes, utilizadas en el mundo, para la Sociedad Española de Salud Ambiental según Casas et al. (2011) son: la Evaluación de Impacto Ambiental definida en la legislación europea, la evaluación de riesgo desarrollada por la Environmental Protection Agency (USA) - Agencia de Protección Ambiental (EPA), la Evaluación de Salud Pública diseñada por la Agency for Toxic Substances and Disease Registry (USA) - Agencia para las Sustancias Tóxicas y el Registro de Enfermedades (ATSDR) y la Evaluación de Impacto en Salud utilizada por la OMS.

Las metodologías mencionadas reconocen al ambiente como agente causal de fenómenos en salud y, aunque cada una aborda el ambiente y su impacto en la salud desde diferentes enfoques investigativos, todas parten de la premisa que define a la salud de una población como el resultado de su interacción con el ambiente; se sustentan en métodos investigativos cuantitativos y cualitativos, con el objetivo de prevenir o revertir los impactos del ambiente en salud. Las que se enfocan en la prevención usan el análisis prospectivo y buscan controlar los factores ambientales y sus posibles efectos, en cambio las que buscan revertir o reparar los daños ya establecidos usan el análisis retrospectivo, estimando impactos ya ocasionados en la salud y los factores ambientales presentes con el objetivo de revertir o cambiar los factores ambientales ya presentes.

El indicador sintético generado en esta investigación obedece a una metodología retrospectiva, analítica, observacional, basada en los impactos ya ocurridos en la población. Estos datos reflejan un comportamiento específico en los municipios del departamento del Meta, caracterizando cada uno de estos según el tipo de evento de morbilidad y mortalidad, estos eventos son considerados por la OMS como efecto de diversos factores ambientales (Ordóñez, 2000; Yassi et al., 2002; Posada et al., 2004).

De acuerdo a los perfiles de morbimortalidad de cada municipio, se infiere la presencia de factores ambientales desencadenantes de la morbimortalidad, reconocidos por la OMS como fuerza impulsora, presión o de exposición en la causalidad de origen ambiental (Yassi et al., 2002; Posada et al., 2004), los cuales son los responsables de los diferentes comportamientos en la degradación en salud ambiental. Además, con la estimación de la morbilidad y la mortalidad se infieren los niveles de necesidad de gestión ambiental (medición del estado y la 
exposición ambiental y la intervención) de cada uno de los municipios, en los factores ambientales relacionados.

De los impactos en salud encontrados en esta investigación, se sugiere la presencia de efectos provenientes de características propias de: el agua (Córdoba et al., 2010; Tobón y López, 2011; Oller-Arlandis y Sanz-Valero, 2012), el aire (De La Rosa et al., 2002; Gavidia et al., 2009; Melgar, 2013), las sustancias químicas (Hernández-Antonio y Hasen, 2011; Valderrama et al., 2012) y la fauna (CruzReyes, 2009; Ramos, 2010), la diferencia en las incidencias y tasas de mortalidad entre los municipios evidencia que cada factor afecta de manera específica cada municipio.

Determinar el estado del ambiente, basado en los efectos de éste en la salud humana es una actividad compleja y costosa, debido al acelerado deterioro ambiental causado por el hombre y las dificultades científicas para medir la exposición de un individuo o de poblaciones a un determinado factor ambiental (Niutta, 1999), además la relación puede verse influida por diversidad de variables como: absorción, distribución, eliminación, la acumulación en el cuerpo, la vía de entrada, la estabilidad de la sustancia química, las interacciones sinérgicas con otras sustancias, la susceptibilidad, los niveles de exposición, las condiciones previas del individuo y la población, etc.

El indicador sintético permite medir la necesidad de gestión ambiental (medición e intervención ambiental) basado en los impactos ocasionados en la salud, ya que su estructuración y construcción se basa en la suma de la mayor cantidad posible de efectos provenientes del ambiente (reflejados como efectos en la salud).

Además, su construcción es de bajo costo al no requerir un sistema propio para la generación de información, debido a que utiliza los datos existentes, recolectados por el sistema de vigilancia en salud pública y la red hospitalaria del Meta.

Los resultados evidencian la existencia de diferencias en los efectos del ambiente sobre la salud en los habitantes de los municipios, permitiendo por consiguiente inferir diferencias en el estado ambiental de los mismos, y convirtiéndolo en un medio eficaz para evaluar el estado de variables ambientales en función de la disponibilidad y facilidad en la obtención de datos (Niutta, 1999).

El indicador se concentra en evidenciar el estado de los efectos, es decir clasifica el estado de salud de los municipios, basado en la morbimortalidad relacionada con variables ambientales, proporciona información acerca de los vínculos entre las condiciones ambientales y la salud como lo hacen otros indicadores de salud ambiental (Kjellström y Corvalán, 1994; Rojas y Guzmán-Piñeiro, 2000; De la Paz et al., 2004) y al igual que estos indicadores la información puede ser utilizada para la gestión de la salud ambiental y la toma de decisiones en gestión ambiental.

\section{CONCLUSIONES}

Existe incidencia y mortalidad en los municipios del departamento de Meta por eventos como: intoxicación por plaguicidas, intoxicación por metales pesados, intoxicación por solventes, intoxicación por sustancias químicas, accidente ofídico, agresiones por animales transmisores de rabia, leptospirosis, enfermedad diarreica aguda, hepatitis A, dengue, malaria, leishmaniasis, enfermedad de Chagas, y enfermedad similar a influenza, para las cuales el ambiente es considerado como determinante en su transmisión u origen, evidenciando que el 
ambiente está ejerciendo efecto sobre la población de los municipios del departamento de Meta.

Este indicador es una herramienta adecuada para la suma de los efectos, ya que permite acumular los efectos en una misma escala de medición y realizar una mejor aproximación al estado ambiental específico para cada factor, logrando mediante éste diferenciar y cuantificar la necesidad de gestión ambiental entre los municipios de Meta.

Se evidenciaron diferencias en los efectos que tienen los factores ambientales sobre la población de los municipios del departamento del Meta, permitiendo concluir que existen grados de afectación en cada uno de los factores para cada municipio.

Es una herramienta económica al usar el sistema de información de vigilancia en salud pública y la red hospitalaria.

\section{RECOMENDACIONES}

Se requiere iniciar procesos de gestión ambiental en todos los municipios del departamento de Meta, por parte de las autoridades y organismos responsables, ya que los indicadores evidencian la existencia de efectos de variables ambientales sobre la salud de sus habitantes.

La academia, los entes territoriales, las autoridades ambientales y de salud deben iniciar el reconocimiento del ambiente como determinante en la salud de sus habitantes, haciéndose partícipes en las decisiones que afectan la calidad ambiental en la que vive su población.

Se debe continuar con el desarrollo de este tipo de indicadores, ejecutándolos en tiempo real para que sirvan como sistema de vigilancia de la salud ambiental y así ser un referente en la toma de decisiones en gestión ambiental.

Los resultados de la presente investigación evidencian la necesidad de construir un diagnóstico ambiental departamental que permita medir y evaluar los factores ambientales como son la calidad del agua, la calidad el aire, la interacción del hombre con la fauna y el uso de las sustancias químicas incluyendo sus características, niveles, fuentes y presiones antrópicas para poder generar intervenciones que mejoren las condiciones ambientales.

Los indicadores generados en esta investigación pueden ser consultados como diagnóstico de la salud ambiental de los municipios del Meta, y ser considerados como la línea base para la construcción de una línea de investigación.

\section{POTENCIAL CONFLICTO DE INTERESES}

En el presente artículo no hay conflicto de intereses. 


\section{FUENTES DE FINANCIACIÓN}

No se tuvo una fuente de financiación.

\section{REFERENCIAS}

- Álvarez-Pérez, A.G., García-Fariñas, A. y Bonet-Gorbea, M. (2007). Pautas conceptuales y metodológicas para explicar los determinantes de los niveles de salud en Cuba. Revista Cubana de Salud Pública, 33(2).

- Casas, S.L., Barbera M., López, E., Aranguez E., Ordóñez J., Martínez, A., Bolo, E. y Escorza F., (2011). La salud en la evaluación de impactos ambientales, guía metodológica. Madrid: Sociedad Española de Sanidad Ambiental. Serie De aeribus, aquis et locis $n^{\circ} 1$.

- Córdoba, M.A., Del Coco, V.F. y Basualdo, J.A. (2010). Agua y salud humana. Revista Química Viva, 3, 117.

- Cruz-Reyes, A. (2009). Fauna feral, fauna nociva y zoonosls. Biodiversidad del ecosistema del Pedregal de San Angel. Sección: restauración, conservación y manejo, 453-461.

- De la Paz, M.P., Carronquino, M.J. y Soldevilla, L. (2004). Indicadores de salud ambiental. Revista de Salud Ambiental, 4(1-2), 1-7.

- De La Rosa, M.d.C., Mosso, M.d.I.A. y Ullán, C. (2002). El aire: hábitat y medio de transmisión de microorganismos. Observatorio Medioambiental, $5,375-402$

- García-Ubaque, C.A., García-Ubaque, J.C. y Vaca-Bohórquez, M.L. (2012). Políticas en salud ambiental, con énfasis en contaminación atmosférica e infancia, en ciudades colombianas. Rev. Salud Pública (Bogotá), 14(S2), 100-112.

- Gavidia, T., Pronczuk, J. y Sly, P. D. (2009). Impactos ambientales sobre la salud respiratoria de los niños: Carga global de las enfermedades respiratorias pediátricas ligada al ambiente. Revista Chilena de Enfermedades Respiratorias, 25, 99-108.

- Hérnández-Antonio, A. y Hasen, A.M. (2011). Uso de plaguicidas en dos zonas agrícolas de México y evaluación de la contaminación de agua y sedimentos. Revista Internacional de Contaminación Ambiental, 27, 115127.

- Instituto Nacional de Salud. (2007). Consolidado Municipal de la vigilancia a la calidad del agua para el consumo humano.

- Kjellström, T. y Corvalán, C. (1994). Framework for the development of environmental health indicators. World health statistics quarterly, 48(2), 144-154.

- Melgar Pérez, J. (2013). Urgencias hospitalarias pediátricas a lo largo de una década: influencia del calendario, condiciones meteorológicas y contaminación atmosférica. Universidad de Oviedo. 
- Ministerio de Ambiente, Vivienda y Desarrollo. (2014). Decreto 1200. Por el cual se determinan los Instrumentos de Planificación Ambiental y se adoptan otras disposiciones.

- Ministerio de Salud y Protección Social. (2012). Dimensiones prioritarias en salud pùblica, Plan decenal de salud pública 2012-2021

- Moiso, A. y Barragán, H. (2007). Determinantes de la salud. Fundamentos de la Salud Pública. La Plata, Argentina: Universidad Nacional de La Plata, Editorial EDULP.

- Niutta, D. (1999). Environmental health indicators. Brown University. Retrieved from http://envstudies.brown.edu/theses/98NIUTTAD.pdf

- Oller-Arlandis, V. y Sanz-Valero, J. (2012). Cáncer por contaminación química del agua de consumo humano en menores de 19 años: una revisión sistemática. Revista Panamericana de Salud Pública, 32, 435443.

- Ordóñez, G. (2000). Salud ambiental: conceptos y actividades. Rev Panam Salud Pública, 7(3), 137-147.

- Organización Panamericana de la Salud. (2001). Informe Regional sobre la Evaluación 2000, en la Región de las Américas: agua potable y saneamiento, estado actual y perspectivas. Retrieved from http://www.bvsde.ops-oms.org/bvsaas/e/fulltext/infregio/infregio.pdf

- Pérez, D., Diago, Y., Corona, B., Espinosa, R. y González, J.E. (2011). Enfoque actual de la salud ambiental. Revista Cubana de Higiene y Epidemiología, 49(1), 84-092.

- Piña, W.A. (2010). Las posibilidades de la evaluación estratégica ambiental integrada: elementos para la reflexión sobre la sostenibilidad urbana en Colombia. Desafíos, 22(2), 181-237.

- Posada, M., Carroquino, J. y Soldevilla, L. (2004). Indicadores de Salud Ambiental. Revista de Salud Ambiental, 4(1-2), 1-7.

- Prüss-Üstün, A. y Corvalán, C.F. (2006). Ambientes saludables y prevención de enfermedades: hacia una estimación de la carga de morbilidad atribuible al medio ambiente. Vol. 1. Ginebra: OMS.

- Puente, C.A. (2008). Evaluación ambiental estratégica para la formulación de política en materia de salud ambiental para Colombia con énfasis en contaminación atmosférica en centros urbanos. Informe ejecutivo final. Universidad Javeriana.

- Quezada, A. (2006). Los objetivos del milenio: algunas tareas para todos. Revista Chilena de Pediatría, 77(2), 123-126.

- Ramos, J.G. (2010). Zoonosis. Gaceta Médica de México, 146, 436-436.

- Rojas, M. y Guzmán-Piñeiro, R. (2000). Indicadores de Salud Ambiental. Ambiente y Saneamiento: Revista Interamericana, 9, 8-13. 
- Schuschny, A. y Soto, H. (2009). Guía metodológica: Diseño de indicadores compuestos de desarrollo sostenible. CEPAL.

- Tobón, M. y López, L. (2011). Genotoxicidad del agua contaminada por plaguicidas en un área de Antioquia. Revista MVZ Córdoba, 16(2).

- Valderrama, F.N., Baena, J.A. y Pérez, J.M. (2012). Persistencia de plaguicidas en el ambiente y su ecotoxicidad: Una revisión de los procesos de degradación natural. Gestión y Ambiente, 15(3), 27-37.

- Villar, E. (2007). Los Determinantes Sociales de Salud y la lucha por la equidad en Salud: desafíos para el Estado y la sociedad civil. Saúde e Sociedade, 16(3), 7-13.

- World Health Organization. (2004). De la teoría a la práctica: indicadores de salud ambiental infantil: implementación de una iniciativa lanzada en la Cumbre Mundial sobre el Desarrollo Sostenible (in press). Ginebra: OMS:

- Yassi, A., Kjellstrom, T., Dekok, T. y Guidotti, T. (2002). Salud ambiental básica. Programa de las Naciones Unidas para el Medio Ambiente Oficina Regional para América y el Caribe.

1. Magíster en Gestión Ambiental Sostenible. Universidad de los Llanos. Villavicencio, Colombia. oagutierrez@unillanos.edu.co

2. Especialista en Finanzas. Universidad de los Llanos. Villavicencio, Colombia. claudia.marcela.martinez@unillanos.edu.co

Para citar este artículo: Gutiérrez Lesmes, O.A. y Martínez Agudelo, C.M. (2016). Indicador sintético para la medición de la necesidad de investigación y gestión ambiental basado en morbimortalidad ocurrida en 2009-2012, Meta, Colombia. Revista Luna Azul, 42, 154-166. Recuperado de vip.ucaldas.edu.co/lunazul/administrator/index.php?option=com_content\&layout $=$ edit\&id $=132$ 